\title{
Púrpura por Strongyloides stercoralis en un paciente inmunocompetente
}

\author{
Raul Herrera', Christian Rojas-Contreras², Gabriel De la Cruz-Ku, \\ Miguel Eduardo Eyzaguirre-Sandovaß,4, Keny Llanos', Carlos Sotelo', \\ Juan Pablo Alcantara', Fabiola Toledo', Milagros Gayoso', Bryan Valcarcel-Valdivia ${ }^{3}$
}

\section{Purpura due to Strongyloides stercoralis in an immunocompetent patient}

Infection with Strongyloides stercoralis is a common parasitic infection in tropical and subtropical regions, including the Peruvian Amazon. The clinical manifestations are varied in patients with immunocompromised disease, and the systemic spread of the disease is frequent, compromising different organs and systems. Cutaneous manifestations are infrequent, being described in patients with some degree of immunosuppression. We present the case of an immunocompetent patient who developed a reactive purpura due to chronic Strongyloides stercoralis infection. Thus, skin involvement is possible in immunocompetent patients with systemic exacerbation due to this parasite.

Key words: Strongyloides stercoralis; strongyloidiasis; purpura; skin manifestations.

Palabras clave: Strongyloides stercoralis; estrongiloidiasis; púrpura; manifestaciones cutáneas.

\section{Introducción}

L a estrongiloidiasis es una parasitosis causada por Strongyloides stercoralis. Se estima que existen 30 a 100 millones de personas infectadas en el mundo ${ }^{1}$. Este parásito se encuentra principalmente en regiones tropicales y subtropicales, como la Amazonía del Perú ${ }^{2}$.

Usualmente, la infección crónica es asintomática. Sin embargo, existen presentaciones leves e inespecíficas, como los síntomas gastrointestinales o la eosinofilia, que persisten por años a pesar que el hospedero ya no resida en áreas endémicas ${ }^{3,4}$. Por otro lado, en pacientes con inmunocompromiso las manifestaciones clínicas son variadas y se presenta frecuentemente como una hiperinfección o infección diseminada, con compromiso de diversos órganos como la piel, corazón, páncreas, vesícula e hígado ${ }^{4,5}$.

En raras ocasiones los pacientes con infección crónica desarrollan síntomas característicos de infección aguda, como las manifestaciones cutáneas, las cuales aún no se encuentran bien caracterizadas ${ }^{6,7}$. Su presentación está asociada a una inmunosupresión y excepcionalmente se manifiestan como lesiones petequiales o purpúricas ${ }^{4,7-9}$. A pesar de que no existe consenso sobre el diagnóstico y el tratamiento, es importante que éstos sean oportunos en la infección crónica para evitar el compromiso sistémico, como el shock séptico ${ }^{10}$.

Se presenta el caso inusual de un paciente inmunocompetente con una infección crónica por Strongyloides stercoralis, que desarrolló una reagudización de la enfermedad, caracterizada por manifestaciones cutáneas.

\section{Caso clínico}

Varón de 20 años de edad, sin antecedentes médicos de importancia, natural y residente de Loreto, Amazonía del Perú. Refirió que hacía nueve meses presentaba lesiones petequiales, eritematosas, circulares, pruriginosas, de bordes bien definidos y localizadas en las piernas, asociado a prurito en la zona plantar que luego se generalizó. Tres meses después inició el servicio militar en Lima, donde se exacerbó el prurito y aumentó el número de lesiones en extremidades superiores e inferiores. Cuatro meses después, se agregó malestar general y fiebre intermitente hasta $40^{\circ} \mathrm{C}$. Acudió a un establecimiento de salud en Rímac, Lima donde se le administró metamizol vía intramuscular. Continuó con esta medicación durante dos meses; sin embargo, ante la no remisión de la fiebre y la progresión de las lesiones consultó en nuestro hospital.

Se admitió al paciente en condiciones generales estables. Al examen físico presentaba lesiones maculopapulares, eritematosas, circulares, confluentes, de bordes bien definidos, de color rojo vinoso, palpables y que no desaparecían a la digitopresión. Tenían un tamaño aproximado de $0,3 \times 0,3 \mathrm{~cm}$ cada una, ubicadas en extremidades superiores e inferiores, tronco, abdomen y región periumbilical, las cuales le producían un prurito intenso. Sin síntomas gastrointestinales.
'Servicio de Medicina Interna del Hospital Militar Central, Lima, Perú.

${ }^{2}$ Servicio de Infectología del Instituto Nacional del Niño-San Borja, Lima, Perú.

${ }^{3}$ Escuela de Medicina Humana en la Universidad Científica del Sur (UCSUR), Lima, Perú.

${ }^{4}$ Sociedad científica de estudiantes de Medicina Humana (SCIEM UCSUR), Lima, Perú.

${ }^{5}$ Facultad de Medicina Humana en la Universidad Ricardo Palma, Lima, Perú.

${ }^{6}$ Facultad de Medicina Humana en la Universidad Privada Antenor Orrego (UPAO), Trujillo, Perú.

Recibido: 11 de diciembre de 2017

Aceptado: 4 de julio de 2018

Correspondencia a: Christian Rojas-Contreras christeorc@gmail.com 


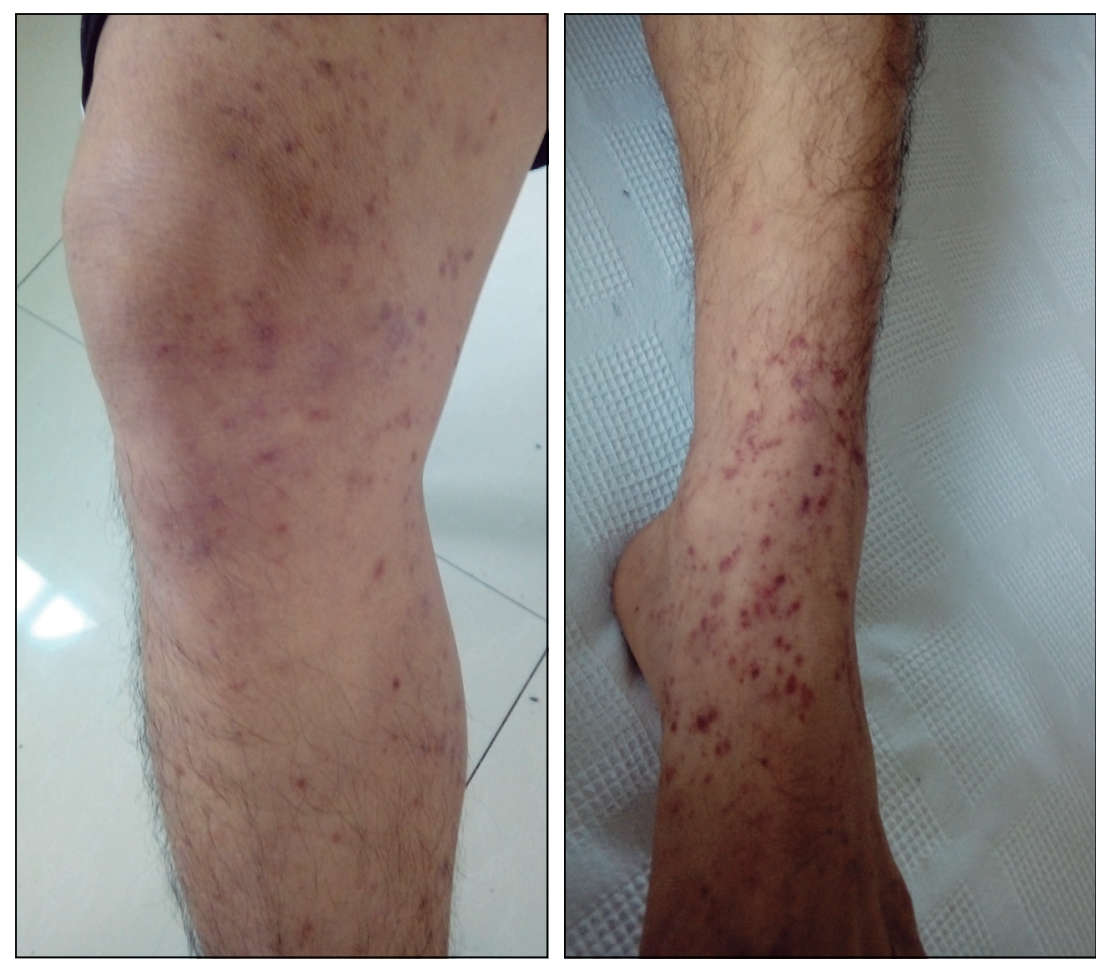

Figura 1. Presencia de pápulas y máculas, eritematosas circulares confluentes de bordes bien definidos, de color rojo vinoso y palpables, en extremidades inferiores.

El hemograma mostró una hipereosinofilia de 50\% (eosinófilos absolutos: 6.480 céls $/ \mathrm{mm}^{3}$ ), con recuento de leucocitos y linfocitos normales. Se plantearon los diagnósticos de una púrpura vascular y una parasitosis inespecífica. Se obtuvo una inmunoglobulina E sérica (IgE) de 609,8 UI/mL, proteína $\mathrm{C}$ reactiva (PCR) de 0,63 $\mathrm{mg} / \mathrm{L}$, y los exámenes serológicos virales (VIH, HTLV-1, VHC, HBsAg) y VDRL resultaron negativos.

Se inició tratamiento sintomático con clorfenamina i.v. Se realizó un examen parasitológico de heces en que se observaron larvas de Strongyloides stercoralis. Se inició ivermectina $200 \mu \mathrm{g} / \mathrm{kg} /$ día, por dos días. Además, se identificó quistes de Entamoeba coli y de Iodamoeba bustchilli; por lo que recibió albendazol $400 \mathrm{mg}$, una vez al día, por siete días. Posteriormente, se administró ivermectina $200 \mathrm{ug} / \mathrm{kg} /$ día por dos días más, a los siete días de la primera dosis.

Luego de 15 días de iniciado el tratamiento, el número de lesiones cutáneas disminuyeron significativamente, aunque mantenían un patrón puntiforme en miembros inferiores y dorso. Por ello se le agregó aceponato de metilprednisolona tópico. Además, en este período, se obtuvo una biopsia de piel la cual identificó un infiltrado linfocitario sin la identificación de larvas. En relación a los exámenes de control, al séptimo día de tratamiento los niveles de eosinófilos descendieron a 24\% (1.685 céls $/ \mathrm{mm}^{3}$ ); y a los 15 días, disminuyeron a $9 \%$ (645 céls/ $\left.\mathrm{mm}^{3}\right)$, mientras la IgE disminuyó a $461 \mathrm{UI} / \mathrm{mL}$.

Al mes del tratamiento, las manifestaciones cutáneas eran escasas. Un examen parasitológico seriado en heces control se observó Hymenolepis nana, por lo que recibió albendazol $400 \mathrm{mg}$ al día por cinco días, prazicuantel 25 $\mathrm{mg} / \mathrm{kg}$ en dosis única y nitazoxanida $500 \mathrm{mg}$, dos veces al día, por tres días. Los niveles de $\operatorname{IgE}$ en un tercer control fue $357,6 \mathrm{UI} / \mathrm{mL}$. Al término del tratamiento, un nuevo examen parasitológico seriado no identificó infestación por parásitos. Finalmente, posterior a dos meses y medio de tratamiento, se dio de alta médica con el diagnóstico de una púrpura reactiva por Strongyloides stercoralis resuelta.

\section{Discusión}

Strongyloides stercoralis es un nemátodo de tierra. Se adquiere principalmente por vía transcutánea a través de microlesiones en el pie ${ }^{5,11}$. Además, el Perú se considera un país altamente endémico por su región amazónica, con una prevalencia de aproximadamente $75 \%$ según los estudios en comunidades ${ }^{2}$. Nuestro paciente provenía de Loreto, departamento localizado en la zona amazónica. Por ello, se considera que adquirió la infección mientras se encontraba residiendo en dicho lugar, probablemente al caminar descalzo en la selva. Esto sugiere una infección crónica, en donde el paciente presentó una reagudización de la misma.

Las presentaciones clínicas se clasifican en agudas, crónicas, el síndrome de hiperinfección y de diseminación. En los hospederos inmunocompetentes, la respuesta celular inmunológica regula el crecimiento de larvas adultas en el intestino; sin embargo, en determinadas ocasiones reaparecen síntomas de infección aguda ${ }^{5,12}$. Por otro lado, los pacientes con inmunosupresión, por enfermedad crónica o en tratamiento con corticoesteroides, desarrollan una hiperinfección y/o diseminación ${ }^{13}$. Las manifestaciones cutáneas en este tipo de pacientes son, en raras ocasiones, petequiales y purpúricas. De este modo, las larvas en la dermis superficial dañan los vasos sanguíneos, causando las petequias. Posteriormente, el consumo plaquetario genera la púrpura ${ }^{4}$. Asimismo, estas lesiones en zona periumbilical se relacionan con un pronóstico desfavorable $e^{4,7-9}$. El paciente no presentaba antecedente de alguna enfermedad crónica inmunosupresora, tratamiento con corticoesteroides u otro fármaco inmunosupresor. No obstante, presentó una manifestación cutánea por $S$. stercoralis, manifestada como púrpura en extremidades superiores e inferiores, tronco, abdomen y región periumbilical. Cabe mencionar que en el estudio histológico de la piel no se identificaron las larvas, probablemente porque la biopsia se pudo efectuar después 
de 15 días del inicio del tratamiento. Se ha descrito la ausencia de larvas en biopsias de piel de pacientes con manifestaciones cutáneas por $S$. stercolaris ${ }^{8,14}$. Además, el hemograma del paciente no mostró leucopenia o linfopenia que pudieran sospechar alguna inmunosupresión. Los exámenes serológicos tampoco detectaron alguna co-infección. La evolución del paciente fue favorable, sin alteración hemodinámica o desarrollo de shock séptico. Efectivamente, se ha descrito que la diseminación puede ocurrir en pacientes inmunocompetentes en ocasiones excepcionales, sin compromiso sistémico que requiera soporte hemodinámico.

Por otro lado, los pacientes con eosinofilia tienen un mejor pronóstico, debido a que la eosinopenia indica algún grado de inmunosupresión ${ }^{5,15}$. El caso actual presentó $50 \%$ de eosinofilia, lo que apoyó a sustentar el estado de inmunocompetencia, y un pronóstico favorable durante el curso de la enfermedad. Además, la elevación de la IgE contribuyó al control inmunitario de la infección, y sus valores disminuyeron conforme el tratamiento se hizo efectivo ${ }^{5}$.

El tratamiento de elección de la estrongiloidiasis es ivermectina, la que se administró en dos ocasiones a nuestro paciente por la no remisión del cuadro. Empero, solo existe evidencia basada en reportes de casos del beneficio del albendazol en la infección diseminada ${ }^{13,16}$. Este fármaco fue parte del tratamiento de nuestro paciente por la infestación con otros parásitos, posiblemente adquiridos durante el servicio militar.

Se concluye que, en pacientes inmunocompetentes, sin factores de riesgo y de zonas endémicas en forma infrecuente pueden desarrollar una reagudización de la infección con diseminación de $S$. stercoralis, asociada a manifestaciones cutáneas. Por ello, el tratamiento oportuno en estos pacientes es esencial, con el fin de evitar la manifestación sistémica y el incremento de morbilidad y mortalidad en los pacientes.

\section{Resumen}

La infección por Strongyloides stercoralis es una parasitosis frecuente en las regiones tropicales y subtropicales, incluyendo la Amazonía peruana. En pacientes con inmunocompromiso, las manifestaciones clínicas son variadas y es frecuente la diseminación sistémica de la enfermedad, con compromiso de diversos órganos. Las manifestaciones cutáneas son infrecuentes y se describen en pacientes con algún grado de inmunosupresión. Se presenta el caso de un paciente inmunocompetente que desarrolló una púrpura reactiva por una infección por Strongyloides stercoralis crónica. Ante ello, es posible el compromiso cutáneo en pacientes inmunocompetentes con reagudización sistémica por este parásito.

\section{Referencias bibliográficas}

1.- Schar F, Giardina F, Khieu V, Muth S, Vounatsou P, Marti H, et al. Occurrence of and risk factors for Strongyloides stercoralis infection in South-East Asia. Acta Trop 2016; 159: 227-38. doi: 10.1016/j. actatropica.2015.03.008.

2.- Schär F, Trostdorf U, Giardina F, Khieu V Muth S, Marti H, et al. Strongyloides stercoralis: global distribution and risk factors. PLoS Negl Trop Dis 2013; 7: e2288. doi: 10.1371/journal.pntd.0002288.

3.- Kalb R E, Grossman M E. Periumbilical purpura in disseminated strongyloidiasis. JAMA 1986; 256: 1170-1.

4.- von Kuster L C, Genta R M. Cutaneous manifestations of strongyloidiasis. Arch Dermatol 1988; 124: 1826-30.

5.- Nutman T B. Human infection with Strongyloides stercoralis and other related Strongyloides species. Parasitology 2017; 144: 263-73. doi: 10.1017/S0031182016 000834.

6.- Arch E L, Schaefer J T, Dahiya A. Cutaneous manifestation of disseminated strongyloidiasis in a patient coinfected with HTLV-I. Dermatol Online J 2008; 14: 6.

7.- $\quad$ Ly M N, Bethel S L, Usmani A S, Lambert D R. Cutaneous Strongyloides stercoralis infection: an unusual presentation. J Am Acad Dermatol 2003; 49 (2 Suppl Case Reports): S157-60.

8.- Salluh J I, Bozza F A, Pinto T S, Toscano L, Weller P F, Soares M. Cutaneous periumbilical purpura in disseminated strongyloidiasis in cancer patients: a pathognomonic feature of potentially lethal disease? Braz J Infect Dis 2005; 9: 419-24.

9.- Martin S J, Cohen P R, MacFarlane D F, Grossman M E. Cutaneous manifestations of Strongyloides stercoralis hyperinfection in an HIV-seropositive patient. Skinmed 2011; 9: 199-202.

10.- Olsen A, van Lieshout L, Marti H, Polderman T, Polman K, Steinmann P, et al. Strongyloidiasis-the most neglected of the neglected tropical diseases? Trans R Soc Trop Med Hyg 2009; 103: 967-72. doi: 10.1016/j. trstmh.2009.02.013.

11.- Greaves D, Coggle S, Pollard C, Aliyu S H, Moore E M. Strongyloides stercoralis infection. BMJ 2013; 347: f4610. doi: 10.1136/bmj.f4610.
12.- van der Feltz M, Slee P H, van Hees P A, Tersmette M. Strongyloides stercoralis infection: how to diagnose best? Neth J Med 1999; 55: 128-31.

13.- Buonfrate D, Requena-Mendez A, Angheben A, Muñoz J, Gobbi F, Van Den Ende J, et al. Severe strongyloidiasis: a systematic review of case reports. BMC Infect Dis 2013; 13: 78. doi: 10.1186/14712334-13-78.

14.- Chen J H, Chao T Y, Perng W C. Strongyloid stercoralis infection of skin and lung. QJM 2010; 103 :199-200. doi: 10.1093/qjmed/ hcp 106.

15.- Ribeiro L C, Rodrigues Junior E N, Silva M D, Takiuchi A, Fontes C J. Púrpura em paciente com estrongiloidíase disseminada. Rev Soc Bras Med Trop 2005; 38: 255-7.

16.- Boggild A K, Libman M, Greenaway C, McCarthy A E, on behalf of the Committee to Advise on Tropical Medicine and Travel (CATMAT). CATMAT statement on disseminated strongyloidiasis: Prevention, assessment and management guidelines. Can Comm Dis Rep 2016; 42: 12-9. https://doi. org/10.14745/ccdr.v42i01a03. 